\title{
POINTWISE ERROR ESTIMATES AND ASYMPTOTIC ERROR EXPANSION INEQUALITIES FOR THE FINITE ELEMENT METHOD ON IRREGULAR GRIDS: PART I. GLOBAL ESTIMATES
}

\author{
ALFRED H. SCHATZ
}

\begin{abstract}
This part contains new pointwise error estimates for the finite element method for second order elliptic boundary value problems on smooth bounded domains in $\mathbb{R}^{N}$. In a sense to be discussed below these sharpen known quasi-optimal $L_{\infty}$ and $W_{\infty}^{1}$ estimates for the error on irregular quasi-uniform meshes in that they indicate a more local dependence of the error at a point on the derivatives of the solution $u$. We note that in general the higher order finite element spaces exhibit more local behavior than lower order spaces. As a consequence of these estimates new types of error expansions will be derived which are in the form of inequalities. These expansion inequalities are valid for large classes of finite elements defined on irregular grids in $\mathbb{R}^{N}$ and have applications to superconvergence and extrapolation and a posteriori estimates. Part II of this series will contain local estimates applicable to non-smooth problems.
\end{abstract}

\section{INTRODUCTION AND DISCUSSION OF RESULTS}

This is the first of a series of papers whose aim is to derive new pointwise error estimates for the finite element method on general quasi-uniform meshes for second order elliptic boundary value problems in $\mathbb{R}^{N}, N \geq 2$. In a sense to be discussed below, these estimates represent an improvement on the now standard quasi-optimal $L_{\infty}$ estimates. In order to fix the ideas, here we will deal with global estimates for a model Neumann problem with smooth solutions. In succeeding papers, local estimates, both interior and up to the boundary, which are applicable to a variety of problems with both smooth and nonsmooth solutions will be considered. As a consequence of these estimates, some new and useful inequalities will be given which are in the form of error expansions. They are valid for large classes of finite elements on general quasi-uniform meshes in $\mathbb{R}^{N}$ and have application to superconvergence and extrapolation and a posteriori esitmates. Let us begin by giving a brief description of some of the main results of this paper.

Let $\Omega$ be a bounded domain in $\mathbb{R}^{N}, N \geq 2$, with smooth boundary $\partial \Omega$. Let

$$
A(u, v)=\int_{\Omega}\left(\sum_{i, j=1}^{N} a_{i j}(x) \frac{\partial u}{\partial x_{i}} \frac{\partial v}{\partial x_{j}}+\sum_{i=1}^{N} b_{i}(x) \frac{\partial u}{\partial x_{i}} v+c(x) u v\right) d x
$$

Received by the editor February 7, 1997.

1991 Mathematics Subject Classification. Primary 65N30.

Supported in part by the National Science Foundation Grant DMS 9403512. 
be coercive over $W_{2}^{1}(\Omega)$ and for given $f \in\left(W_{2}^{1}(\Omega)\right)^{\prime}$ let $u \in W_{2}^{1}(\Omega)$ be the solution of the Neumann problem with homogeneous boundary data defined by

$$
A(u, v)=(f, v)=\int_{\Omega} f v d x \quad \text { for all } v \in W_{2}^{1}(\Omega) .
$$

It is well known that if $f$ is smooth in $\bar{\Omega}$, then $u$ is also.

Now consider the approximation of $u$ using the finite element method. Let $0<h<1$ be a parameter, $r \geq 2$ be an integer and $S_{r}^{h}(\Omega) \subset W_{\infty}^{1}(\Omega)$ be a family of finite element spaces. The precise assumptions on these subspaces are given in Section 1 and are satisfied by many types of commonly used finite elements. For the purposes of this introduction they may be thought of as any one of a variety of spaces of continuous functions, which on each set $\tau$ of a quasi-uniform partition of $\Omega$, roughly of size $h$, contains all polynomials of degree $r-1$ and fit the boundary exactly. For example $r=2$ could correspond to piecewise linear (or bilinear, etc.) functions and $r=3$ to piecewise quadratic functions, etc. Thus they can approximate functions to order $h^{r}$ in $L_{\infty}(\Omega)$ and order $h^{r-1}$ in $W_{\infty}^{1}(\Omega)$. The finite element approximation $u_{h} \in S_{r}^{h}(\Omega)$ is taken to satisfy

$$
A\left(u_{h}, \varphi\right)=(f, \varphi) \quad \text { for all } \varphi \in S_{r}^{h}(\Omega)
$$

or

$$
A\left(u-u_{h}, \varphi\right)=0 \quad \text { for all } \varphi \in S_{r}^{h}(\Omega) .
$$

Quasi-optimal $L_{\infty}$ estimates on general quasi-uniform meshes for the finite element method were first proved by Natterer [3] and Scott [13] in 1975. These were followed by many other studies which refined and extended their results to more general situations (see for example [4], [5], [7], [8], [9], [10] and [11] to name a few). These estimates take the form

$$
\left\|u-u_{h}\right\|_{L_{\infty}(\Omega)} \leq C h\left(\ln \frac{1}{h}\right)^{\bar{r}} \inf _{\chi \in S_{r}^{h}}\|u-\chi\|_{W_{\infty}^{1}(\Omega)}
$$

and

$$
\left\|u-u_{h}\right\|_{W_{\infty}^{1}(\Omega)} \leq C \inf _{\chi \in S_{R}^{h}}\|u-\chi\|_{W_{\infty}^{1}(\Omega)} .
$$

In (0.5) $\bar{r}=1$ if $r=2$ and $\bar{r}=0$ if $r \geq 3$. The constants $C$ in (0.5) and (0.6) are independent of $u, u_{h}$ and $h$.

The results derived in this paper start with a slightly different point of view. They are based on the fact that part of all of the present proofs of the global $L_{\infty}$ estimates have much in common with the proofs of local $L_{2}$ based error estimates, where cut-off functions are replaced by weight functions. The proofs in a sense are local in nature. Hence here we shall focus our attention not on the $L_{\infty}(\Omega)$ or $W_{\infty}^{1}(\Omega)$ norm of the error but rather on the error at an arbitrary but fixed point $x$ of $\bar{\Omega}$.

In order to describe our first results we shall need some notation. For each fixed point $x \in \bar{\Omega}$, real number $s$ and arbitrary $y \in \mathbb{R}^{N}$ consider the weight function

$$
\sigma_{x, h}^{s}(y)=\left(\frac{h}{|x-y|+h}\right)^{s} .
$$

Notice that if $s>0$ and $|x-y|=O(h)$ then $\sigma_{x}^{s}(y)=0(1)$. Furthermore $\sigma_{x}^{s}(y)$ is a decreasing function of $|x-y|$ and $\sigma_{x}^{s}(y)=O\left(h^{s}\right)$ when $|x-y|=O(1)$. A plot of $\sigma_{x}^{s}(y)$ vs. $|x-y|$ is given in Figure 1. 


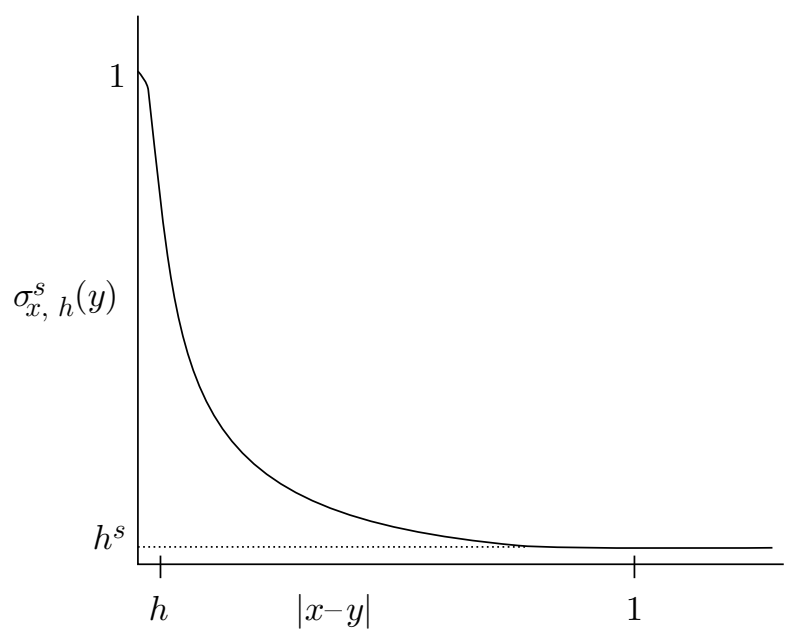

FiguRE 1

Remark. The denominator in (0.7) can be replaced with the "equivalent" $\left(|x-y|^{2}+h^{2}\right)^{1 / 2}$ without effecting the results to follow.

For $1 \leq p \leq \infty$ and fixed $x$ consider the weighted norms

$$
\|u\|_{L_{p}(\Omega), x, s}=\left\|\sigma_{x}^{s}(y) u(y)\right\|_{L_{p}(\Omega)}
$$

and

$$
\|u\|_{W_{p}^{1}(\Omega), x, s}=\|u\|_{L_{p}(\Omega), x, s}+\|\nabla u\|_{L_{p}(\Omega), x, s} .
$$

Notice that if $p=\infty$ and $s=0$ these weighted norms satisfy, for continuous $u$,

$$
|u(x)| \leq\|u\|_{L_{\infty}(\Omega), x, s} \leq\|u\|_{L_{\infty}(\Omega)}
$$

and at points where $\nabla u(x)$ is continuous

$$
|\nabla u(x)| \leq\|u\|_{W_{\infty}^{1}(\Omega), x, s} \leq\|u\|_{W_{\infty}^{1}(\Omega)} .
$$

Our first result concerns the error of $\left(u-u_{h}\right)(x)$ at an arbitrary but fixed point $x \in \bar{\Omega}$ and is given in Theorem 2.1, which may be roughly stated as follows: Let $x \in \bar{\Omega}$ and $0 \leq s \leq r-2$, then

$$
\left|\left(u-u_{h}\right)(x)\right| \leq\left\|u-u_{h}\right\|_{L_{\infty}(\Omega), x, s} \leq C h\left(\ln \frac{1}{h}\right)^{\bar{s}} \inf _{\chi \in S_{r}^{h}}\|u-\chi\|_{W_{\infty}^{1}(\Omega), x, s} .
$$

Here $\bar{s}=1$ if $s=r-2, \bar{s}=0$ if $0 \leq s<r-2$ and $C$ is independent of $u, u_{h}$ and $x$.

It is easy to see that $(0.12)$ is sharper than (0.5) when $r \geq 3$. In fact choosing $x \in \bar{\Omega}$ to be the point where $\left|\left(u-u_{h}\right)(x)\right|=\left\|u-u_{h}\right\|_{L_{\infty}(\Omega)}$ and using the inequality (0.11), it follows that (0.12) implies (0.5) when $0<s<r-2$ but not vice versa. The estimate (0.12) gives new information about the behavior of the error at a fixed but arbitrary point $\bar{x} \in \Omega$. Because of the weighted norms on the right, it indicates a more local dependence of the error at $x$ on the solution $u$ in a neighborhood of $x$ than is indicated by (0.5). Furthermore the larger the $r$ the more local that dependence is. 
Remark. Under some additional assumptions on the subspaces (see the Remark 2.1 after Theorem 2.2), the term

$$
h \inf _{\chi \in S_{r}^{h}}\|u-\chi\|_{W_{\infty}^{1}(\Omega), x, s}
$$

may be replaced by

$$
\inf _{\chi \in S_{r}^{h}}\|u-\chi\|_{L_{\infty}(\Omega), x, s} .
$$

Let us briefly discuss the differences in the proofs of (0.5) and (0.12). The starting point that can be used for both is the representation

$$
\left|\left(u-u_{h}\right)(x)\right| \leq\left|A\left(u-\chi, g^{x}-g_{h}^{x}\right)\right|
$$

where $g^{x}$ may be thought of as a "smoothed" Green's function with singularity at $x$, $g_{h}^{x} \in S_{r}^{h}(\Omega)$ is its finite element approximation and $\chi \in S_{r}^{h}(\Omega)$ is arbitrary. So the problem reduces to obtaining estimates for $g^{x}-g_{h}^{x}$. An analogous approach has been used previously for obtaining pointwise estimates for finite difference methods and was also used by Scott [13] in analyzing the finite element method. The estimate (0.5) for the $L_{\infty}$ norm follows by taking

$$
\left\|u-u_{h}\right\|_{L_{\infty}(\Omega)} \leq C\|u-\chi\|_{W_{\infty}^{1}(\Omega)} \sup _{x \in \bar{\Omega}}\left\|g^{x}-g_{h}^{x}\right\|_{W_{1}^{1}(\Omega)}
$$

and showing that

$$
\sup _{x \in \bar{\Omega}}\left\|g^{x}-g_{h}^{x}\right\|_{W_{1}^{1}(\Omega)} \leq C h\left(\ln \frac{1}{n}\right)^{\bar{r}} .
$$

If one thinks of $g^{x}$ as "almost" being in $W_{1}^{2}(\Omega)$, then (0.14) is reasonable from the point of view of approximation theory, in terms of powers of $h$. This type of estimate is in fact proved in all those papers using this approach. On the other hand if one thinks of the Green's function with singularity at $x$, its "nonsmooth" behavior occurs only at $x$. Away from $x$ it satisfies a homogeneous elliptic equation and hence not only is it smooth but its derivatives have very special decay properties as a function of inverse powers of the distance to the singularity. Thus we might hope that away from $x, g^{x}$ may be approximated to order $h^{r-1}$ in $W_{1}^{1}$ by using the fact that it is in $W_{1}^{r}$ and then bound the $r^{\text {th }}$ order derivatives in terms of inverse powers of the distance to $x$. This in fact can be done and we shall prove the weighted estimate

$$
\left\|g^{x}-g_{h}^{x}\right\|_{W_{1}^{1}(\Omega), x,-s} \leq C h\left(\ln \frac{1}{h}\right)^{\bar{s}}
$$

where $\bar{s}=0$ for $0 \leq s<r-2, \bar{s}=1$ if $s=r-2$ and $C$ is independent of $h$ and $x$. The presence of the weight $\sigma_{x}^{-s}(y)$ indicates that the estimate $(0.15)$ is in general stronger than (0.14).

Interior pointwise error estimates for the Green's function for this problem were proved in Schatz and Wahlbin [9]. The estimate (0.12) now follows from (0.15) and (0.13) which can be estimated by

$$
\left|\left(u-u_{h}\right)(x)\right| \leq C\|u-\chi\|_{W_{\infty}^{1}(\Omega), x, s}\left\|g^{x}-g_{h}^{x}\right\|_{W_{1}^{1}(\Omega), x,-s} .
$$

There is an analogous result for pointwise error estimates for first derivatives which is given in Theorem 3.1. This may be roughly stated as follows: Let $x \in \bar{\Omega}$ 
and $0 \leq s \leq r-1$, then

$$
\left\|u-u_{h}\right\|_{W_{\infty}^{1}(\Omega), x, s} \leq C\left(\ln \frac{1}{h}\right)^{\overline{\bar{s}}} \inf _{\chi \in S_{r}^{h}}\|u-\chi\|_{W_{\infty}^{1}(\Omega), x, s} .
$$

Here $\overline{\bar{s}}=0$ if $0 \leq s<r-1, \overline{\bar{s}}=1$ if $s=r-1$ and $C>0$ is independent of $u, u_{h}$, $h$, and $x$.

We remark that the proofs of Theorem 2.1 and Theorem 3.1 have much in common with the proofs of results given in Schatz and Wahlbin [9] and [10].

In view of our previous discussion it is easily seen that (0.16) implies (0.6), for $0<s<r-1$ but not vice versa. Hence (0.16) is sharper than (0.6) this time for $r \geq 2$, and also because the weighted norm on the right indicates a far more local dependence of derivatives of the error on $u$ than is indicated by (0.6).

In this direction one consequence of the weighted estimates $(0.12)$ and $(0.16)$ are estimates that we shall call "error expansion inequalities". They show the local dependence of the error on $u$. There are many variations which are easily derived from (0.12) and (0.16). Here we shall present a special case of a result given in Theorem 3.1. We begin with estimates for $\left(u-u_{h}\right)(x)$.

Suppose $r \geq 3$ and $u \in W_{\infty}^{2 r-2}(\Omega)$, then there exists a constant $C$ independent of $u, u_{h}, h$ and $x$ such that

$$
\begin{aligned}
& \left|\left(u-u_{h}\right)(x)\right| \\
& \leq C\left(\ln \frac{1}{h}\right)\left(h^{r} \sum_{|\alpha|=r}\left|D^{\alpha} u(x)\right|+\cdots+h^{2 r-3} \sum_{|\alpha|=2 r-3}\left|D^{\alpha} u(x)\right|\right. \\
& \left.\quad+h^{2 r-2}\|u\|_{W_{\infty}^{2 r-2}(\Omega)}\right) .
\end{aligned}
$$

A corresponding estimate for derivatives is as follows: Suppose $r \geq 2$ and $u \in$ $W_{\infty}^{2 r-1}(\Omega)$, then there exists a constant $C$ independent of $u, u_{h}, h$ and $x$ such that

$$
\begin{aligned}
& \left|\nabla\left(u-u_{h}\right)(x)\right| \\
& \leq C\left(\ln \frac{1}{h}\right)\left(h^{r-1} \sum_{|\alpha|=r}\left|D^{\alpha} u(x)\right|+\cdots+h^{2 r-3} \sum_{|\alpha|=2 r-2}\left|D^{\alpha} u(x)\right|\right. \\
& \left.\quad+h^{2 r-2}\|u\|_{W_{\infty}^{2 r-1}(\Omega)}\right) .
\end{aligned}
$$

We remark that these inequalities may be trivially changed to equalities with the constants $C$ replaced by functions $g\left(x, u, u_{h}, h\right) \geq 0$, which depend on $x, u, u_{h}$, and $h$ such that $g\left(x, u, u_{h}, h\right) \leq C$ independent of $x, u, u_{h}$ and $h$.

Notice that all the terms on the right except the last in $(0.17)$ and $(0.18)$ involve derivatives of $u$ at only one point. We would like to emphasize again that these expansions are valid at any point of $\bar{\Omega}$ and for a large class of finite elements in $\mathbb{R}^{N}, N \geq 2$, and for equations of the form $(0.1),(0.2)$. Other expansions will be given in forthcoming papers for different problems. With regard to other work, a precise asymptotic expansion has been derived in Blum, Lin and Rannacher [1] for the special case of Dirichlet's problem in the plane for $-\Delta u=f$ in $\Omega, u=0$ on $\partial \Omega$. Their expansions are valid at special points $x$ on a "two regular grid" using piecewise linear elements. The approach used there is entirely different and seems not to easily generalize to more general situations. 
We end this introduction by mentioning some consequences of the estimates (0.17) and (0.18) given in Corollaries 4.1 through 4.3. Very roughly stated, Corollaries 4.1 and 4.2 say respectively that if $D^{\alpha} u(x)=0$ for all $|\alpha|=r$, then the rate of convergence of $\left(u-u_{h}\right)(x)$ for $r \geq 3$ is greater than $h^{r}$ and when $r \geq 2$ the rate of convergence of $\nabla\left(u-u_{h}\right)(x)$ is greater than $h^{r-1}$. In Corollary 4.3 we shall give a sufficient condition on $u$ such that the error at a point may be bounded above by the local interpolation error. In a future publication we shall use local results of this type together with some additional ideas to obtain some new superconvergence and extrapolation results for the finite element method and investigate some pointwise a posteriori error estimators (cf. [14]).

A brief outline of this paper is as follows: In Section 1 we discuss some preliminaries. Section 2 contains results on pointwise estimates and Section 3 estimates for derivatives. Section 4 contains results on error expansion inequalities.

\section{Preliminaries}

(A) A Neumann problem. Let $\Omega$ be a bounded domain in $\mathbb{R}^{N}$ with smooth boundary $\partial \Omega$. For any domain $D \subseteq \Omega, t \geq 0$ an integer and $1 \leq p \leq \infty, W_{p}^{t}(D)$ and $\stackrel{\circ}{W}_{p}^{t}(D)$ will denote the usual Sobolev spaces with the usual norms $\|\cdot\|_{W_{p}^{t}(D)}$. For $t<0$ and $1 \leq p \leq \infty, W_{p}^{t}(D)$ will denote the dual of $\stackrel{\circ}{W}_{q}^{-t}(D)$ with the norm

$$
\|u\|_{W_{p}^{t}(D)}=\sup _{\substack{v \in C_{0}^{\infty}(\Omega) \\ v \neq 0}} \frac{\int_{D} u v d x}{\|v\|_{W_{q}^{-t}(D)}}, \quad \frac{1}{p}+\frac{1}{q}=1 .
$$

$\widetilde{W}_{p}^{t}(D)$ will denote the dual of $W_{q}^{-t}(D)$.

Consider the Neumann problem with homogeneous boundary conditions

$$
\begin{gathered}
L u=-\sum_{i, j=1}^{N} \frac{\partial}{\partial x_{j}}\left(a_{i j}(x) \frac{\partial u}{\partial x_{i}}\right)+\sum_{i=1}^{N} b_{i}(x) \frac{\partial u}{\partial x_{i}}+C(x) u=f \text { in } \Omega \\
\frac{\partial u}{\partial n_{L}}=0 \quad \text { on } \quad \partial \Omega
\end{gathered}
$$

where $\frac{\partial u}{\partial n_{L}}$ denotes the co-normal derivative on $\partial \Omega$. For simplicity we shall assume that the coefficients $a_{i j}, b_{i}$ and $c$ are in $C^{\infty}(\bar{\Omega})$. These conditions can be weakened (see Remark 1.1). Furthermore assume that $L$ is uniformly elliptic, i.e., there exists an $m_{0}>0$ such that

$$
m_{0} \sum_{i=1}^{N} \zeta_{i}^{2} \leq \sum_{i, j=1}^{N} a_{i j}(x) \zeta_{i} \zeta_{j} \quad \text { for all } \quad \zeta \in \mathbb{R}^{N} \quad \text { and } \quad x \in \Omega .
$$

The weak formulation of $(1.2),(1.3)$ is given in $(0.2)$ where $A(\cdot, \cdot)$ is defined by (0.1). Throughout this paper it will be assumed that $A(\cdot, \cdot)$ is coercive on $W_{2}^{1}(\Omega)$, i.e., there exists an $m>0$ such that

$$
m\|u\|_{W_{2}^{1}(\Omega)}^{2} \leq A(u, u) \text { for all } u \in W_{2}^{1}(\Omega) .
$$

In this case both $(0.2)$ and its adjoint problem

$$
A(v, w)=(f, v) \text { for all } \quad v \in W_{2}^{1}(\Omega)
$$


have unique solutions for each $f \in \widetilde{W}_{2}^{-1}(\Omega)$. If $f \in W_{p}^{t}(\Omega)$ for $t$ an integer $0 \leq t \leq$ $r-2$ and $2 \leq p<\infty$, then $u$ and $w \in W_{p}^{t+r}(\Omega)$ and

$$
\begin{aligned}
\|u\|_{W_{p}^{t+2}(\Omega)} & \leq C p\|f\|_{W_{p}^{t}(\Omega)}, \\
\|w\|_{W_{p}^{t+2}(\Omega)} & \leq C p\|f\|_{W_{p}^{t}(\Omega)},
\end{aligned}
$$

where $C$ is independent of $f, t$ and $p$.

Let us remark that as discussed in Schatz and Wahlbin [10], the dependence of (1.7) on $p$ for $t=2$ may be found by tracing constants in, for example, the proof given in Gilbarg and Trudinger [15]. The estimate for higher derivatives may be found by bootstrapping with that case.

Let $G^{x}(y)$ denote the Green's function for the problem (1.2), (1.3) with singularity at $x$. It will be convenient to use the following estimates for $G^{x}(y)$ which can be found in Krasovskii [2].

Lemma 1.1. There exists a constant $C$ such that for $x, y \in \bar{\Omega}$

$$
\left|D_{x}^{\alpha} D_{y}^{\beta} G^{x}(y)\right| \leq C|x-y|^{2-N-|\alpha+\beta|} \text { for } \quad|\alpha+\beta|>0 .
$$

Here $C$ depends only on $\Omega, m_{0}, m$ and various norms of the coefficients.

Remark 1.1. If $r \geq 2$ is an integer, then (1.8) holds for $0<|\alpha+\beta| \leq r$ if for example $a_{i j}, b_{i}, c \in C^{r+1}$ and $\Omega \in C^{r+3}$.

(B) The finite element subspaces. We shall now state our assumptions on the finite element spaces used in this paper. They are basically, with some slight simplifications, the same as those given in Schatz and Wahlbin [10] and [11].

For $0<h<1$ a parameter and $r \geq 2$ an integer, $S_{r}^{h}(\Omega)$ will denote a family of finite dimensional subspaces of $W_{\infty}^{1}(\Omega)$. If $D \subseteq \Omega$, then $S_{r}^{h}(D)$ will denote the restriction of functions in $S_{r}^{h}(\Omega)$ to $D$. In what follows $\widetilde{D}_{0} \subset \subset \widetilde{D}_{1} \subset \subset \widetilde{D}_{2}$ will denote concentric balls and $D_{i}=\widetilde{D}_{i} \cap \Omega, i=0,1,2$.

Assume that there exist a constant $k$ such that if $\operatorname{dist}\left(\widetilde{D}_{0}, \partial \widetilde{D}_{1}\right) \geq k h$ and $\operatorname{dist}\left(\widetilde{D}_{1}, \partial \widetilde{D}_{2}\right) \geq k h$, then the following hold:

A.1 (Approximation). If $t=0,1, t \leq \ell \leq r, 1 \leq p \leq \infty$, then for each $v \in W_{p}^{\ell}\left(D_{2}\right)$ there exists a $\chi \in S_{r}^{h}\left(D_{2}\right)$ such that

$$
\|v-\chi\|_{W_{p}^{t}\left(D_{1}\right)} \leq C h^{\ell-t}\|v\|_{W_{p}^{\ell}\left(D_{2}\right)} .
$$

If $N<p \leq \infty$

$$
\|v-\chi\|_{W_{\infty}^{1}\left(D_{1}\right)} \leq C h^{r-1-N / p}\|v\|_{W_{p}^{r}\left(D_{2}\right)} .
$$

Furthermore if $v$ vanishes outside of $D_{0}, \chi$ vanishes outside of $D_{1}$. The constant $C$ is independent of $h, v, \chi, D_{1}$ and $D_{2}$.

A.2 (Inverse properties). If $\chi \in S_{r}^{h}(\Omega)$, then for $t=0,1$ and $\ell \geq 0$ is an integer and $1 \leq q \leq p \leq \infty$,

$$
\|\chi\|_{W_{\infty}^{p}\left(D_{1}\right)} \leq C h^{-\left[\frac{N}{q}-\frac{N}{p}\right]-t-\ell}\|\chi\|_{W_{q}^{-\ell}\left(D_{2}\right)} .
$$

For easy reference we single out the special cases of (1.10) where for $\ell=0,1$

$$
\|\chi\|_{W_{2}^{t}\left(D_{1}\right)} \leq C h^{\ell-t}\|\chi\|_{W_{2}^{-\ell}\left(D_{2}\right)} .
$$

Here $C$ is independent of $h, \chi, D_{1}$ and $D_{2}$. 
A.3 (Superapproximation). Let $\omega \in C_{0}^{\infty}\left(\widetilde{D}_{1}\right)$, then for each $\chi \in S_{r}^{h}\left(D_{3}\right)$ there exists an $\eta \in S_{r}^{h}\left(D_{3}\right)$, vanishing outside of $D_{2}$, such that for some integer $\gamma>0$

$$
\|\omega \chi-\eta\|_{W_{2}^{1}\left(D_{3}\right)} \leq C h\|\omega\|_{W_{\infty}^{\gamma}\left(D_{1}\right)}\|\chi\|_{W_{2}^{1}\left(D_{3}\right)} .
$$

Furthermore, if $\omega \equiv 1$ on $\widetilde{D}_{0}$ and $\widetilde{D}_{-1} \subset \subset \widetilde{D}_{0}$ with $\operatorname{dist}\left(\widetilde{D}_{-1}, \partial \widetilde{D}_{0}\right) \geq k$, then $\eta=\chi$ on $D_{-1}$ and

$$
\|\omega \chi-\eta\|_{W_{2}^{1}\left(D_{2}\right)} \leq C h\|\omega\|_{W_{\infty}^{\gamma}\left(D_{1}\right)}\|\chi\|_{W_{2}^{1}\left(D_{2} \backslash D_{0}\right)} .
$$

Here $C$ is independent of $\omega, \chi, \eta, h, D_{0}, D_{1}$ and $D_{2}$.

A.4 (Scaling). Let $x_{0} \in \bar{\Omega}$ and $d \geq k h$. The linear transformation $y=\left(x-x_{0}\right) / d$ takes $B_{d}\left(x_{0}\right)=\left\{x:\left|x-x_{0}\right|<d\right\} \cap \Omega$ into a new domain $\widehat{B}_{1}\left(x_{0}\right)$ and $S_{r}^{h}\left(B_{d}\left(x_{0}\right)\right)$ into a new function space $\widehat{S}_{r}^{h / d}\left(\widehat{B}_{1}\left(x_{0}\right)\right)$. Then $\widehat{S}_{r}^{h / d}\left(\widehat{B}_{1}\left(x_{0}\right)\right)$ satisfies A.1, A.2 and A.3 with $h$ replaced by $h / d$. The constants occurring in A.1, A.2 and A.3 remain unchanged, in particular independent of $d$.

(C) Some preliminary error estimates. For $v \in W_{2}^{1}(\Omega)$ let $v_{h}$ be either the solution of

$$
A\left(v-v_{h}, \varphi\right)=0 \quad \text { for all } \quad \varphi \in S_{r}^{h}(\Omega)
$$

or the solution of the adjoint problem

$$
A\left(\varphi, v-v_{h}\right)=0 \quad \text { for all } \varphi \in S_{r}^{h}(\Omega) .
$$

We shall need two well known error estimates, one global and one local, for the problems (1.14) and (1.15). First a well-known global estimate.

Lemma 1.2. Let $v$ and $v_{h}$ be as above, then

$$
\left\|v-v_{h}\right\|_{L_{2}(\Omega)}+h\left\|v-v_{h}\right\|_{W_{2}^{1}(\Omega)} \leq C h\|f\|_{L_{2}(\Omega)} .
$$

We shall state the local results for special subdomains of $\Omega$. Without loss of generality we may assume throughout this paper that diam $(\Omega) \leq 1$. Let

$$
d_{j}=2^{-j} \quad \text { for } \quad j=0,1,2, \ldots
$$

and for fixed $x$ set

$$
\begin{aligned}
& \Omega_{j}=\left\{y \in \Omega: d_{j+1}<|y-x|<d_{j}\right\}, \\
& \Omega_{j}^{\prime}=\left\{y \in \Omega: d_{j+2}<|y-x|<d_{j-1}\right\}, \\
& \Omega_{j}^{\prime \prime}=\left\{y \in \Omega: d_{j+3}<|y-x|<d_{j-2}\right\} .
\end{aligned}
$$

Lemma 1.3. Suppose that A.1-A.4 are satisfied and that $v-v_{h}$ satisfies either (1.14) and (1.15). Suppose that $0 \leq t \leq r-2$ is an integer, then if $d_{j} \geq k h$

$$
\left\|v-v_{h}\right\|_{W_{2}^{1}\left(\Omega_{j}\right)} \leq C\left(h^{r-1}\|v\|_{W_{2}^{r}\left(\Omega_{j}^{\prime}\right)}+d_{j}^{-N / 2-1-t}\left\|v-v_{h}\right\|_{W_{1}^{-t}\left(\Omega_{j}^{\prime}\right)}\right),
$$

where $C$ is independent of $v, v_{h}, h$ and $j$.

If $\Omega_{j}$ is an interior subdomain of $\Omega$, then the result can be found in Schatz and Wahlbin [11] and for domains abutting the boundary, it can be found in Schatz and Wahlbin [10]. They are adaptations of the local result given in Nitsche and Schatz [6].

We shall now define two functions $g^{x}(y)$ and $g_{h}^{x}(y) \cdot g^{x}(y)$ may be thought of as a smoothed Green's function with singularity at $x$, and $g_{h}^{x}(y) \in S_{r}^{h}$ its finite 
element approximation. We now give some facts that will be needed for the proof of pointwise estimates.

For $d>0$ and any fixed $x \in \bar{\Omega}, B_{d}(x)$ will denote the intersection of $\Omega$ with a ball of radius $d$ centered at $x$, i.e.,

$$
B_{d}(x)=\{y \in \Omega:|y-x|<d\} .
$$

Let $k$ be as in A.1-A.4 and $u-u_{h}$ satisfy (1.14). Define

$$
\eta(y)=\left\{\begin{array}{cl}
h^{-N / 2}\left(u-u_{h}\right)(y) /\left\|u-u_{h}\right\|_{L_{2}\left(B_{2 k h}(x)\right)} & \text { for } y \in B_{2 k h}(x), \\
0 & \text { elsewhere. }
\end{array}\right.
$$

Notice that $\operatorname{supp}(\eta) \subseteq \overline{B_{2 k h}(x)}$ and $\|\eta\|_{L_{2}\left(B_{2 k h}(x)\right)}=h^{-N / 2}$. For fixed $x \in \bar{\Omega}, g^{x}(y)$ is defined to satisfy

$$
A\left(v, g^{x}\right)=(\eta, v) \quad \text { for all } v \in W_{2}^{1}(\Omega) .
$$

The finite element approximation $g_{h}^{x}(y) \in S_{r}^{h}(\Omega)$ is taken to be the unique solution of

$$
A\left(\varphi, g^{x}-g_{h}^{x}\right)=0 \quad \text { for all } \varphi \in S_{r}^{h}(\Omega) .
$$

The importance of $g^{x}$ and $g_{h}^{x}$ is the following:

Lemma 1.4. Let $u-u_{h}$ satisfy (1.14) and $g^{x}-g_{h}^{x}$ satisfy (1.21), then for any fixed $x \in \bar{\Omega}, 0 \leq s \leq s_{0}$ for any fixed $s_{0}$ and any $\chi \in S_{r}^{h}(\Omega)$

$$
\left|\left(u-u_{h}\right)(x)\right| \leq C\left(\left\|g^{x}-g_{h}^{x}\right\|_{W_{1}^{1}(\Omega), x,-s}+h\right)\|u-\chi\|_{W_{\infty}^{1}(\Omega), x, s} .
$$

Here $C$ is independent of $u, u_{h}, g^{x}, g_{h}^{x}, h, x, s$ and $\chi$.

Proof. For any $\psi \in S_{r}^{h}(\Omega), 0 \leq s \leq s_{0}$, the triangle inequality A.1 and A.2 yield

$$
\begin{aligned}
& \left|\left(u-u_{h}\right)(x)\right| \leq|(u-\psi)(x)|+C h^{-N / 2}\left\|\psi-u_{h}\right\|_{L_{2}\left(B_{2 k h}(x)\right)} \\
& \quad \leq|(u-\psi)(x)| \\
& \quad+C h^{-N / 2}\left(\|\psi-u\|_{L_{2}\left(B_{2 k h}(x)\right)}+\left\|u-u_{h}\right\|_{L_{2}\left(B_{2 k h}(x)\right)}\right) \\
& \quad \leq C(k)\|u-\psi\|_{L_{\infty}\left(B_{2 k h}(x)\right)}+C h^{-N / 2}\left\|u-u_{h}\right\|_{L_{2}\left(B_{2 k h}(x)\right)} \\
& \leq C(k) h\|u\|_{W_{\infty}^{1}\left(B_{3 k h}(x)\right)}+C h^{-N / 2}\left\|u-u_{h}\right\|_{L_{2}\left(B_{2 k h}(x)\right)} \\
& \leq C(k) h\|u\|_{W_{\infty}^{1}(\Omega), s, x}+C h^{-N / 2}\left\|u-u_{h}\right\|_{L_{2}\left(B_{2 k h}(x)\right)} .
\end{aligned}
$$

In view of (1.20), (1.21) and (0.4)

$$
\begin{aligned}
h^{-N / 2}\left\|u-u_{h}\right\|_{L_{2}\left(B_{2 k h}(x)\right)} & =\left(u-u_{h}, \eta\right)=A\left(u-u_{h}, g^{x}\right) \\
& =A\left(u-u_{h}, g^{x}-g_{h}^{x}\right)=A\left(u, g^{x}-g_{h}^{x}\right) \\
& \leq C\left\|g^{x}-g_{h}^{x}\right\|_{W_{1}^{1}(\Omega), x-s}\|u\|_{W_{\infty}^{1}(\Omega), x, s} .
\end{aligned}
$$

This inequality together with (1.23) yields

$$
\left|\left(u-u_{h}\right)(x)\right| \leq C\left(\left\|g^{x}-g_{h}^{x}\right\|_{W_{1}^{1}(\Omega), x,-s}+h\right)\|u\|_{W_{\infty}^{1}(\Omega), x, s} .
$$

The inequality (1.22) now follows by applying (1.24) with $(u-\chi)-\left(u_{h}-\chi\right)$, for any $\chi \in S_{r}^{h}(\Omega)$, in place of $u-u_{h}$, which completes the proof. 
In the next section we shall prove the weighted estimate (0.15) for $g^{x}-g_{h}^{x}$ using the local estimate (1.18) and the global estimate (1.16) as primary tools. Here we collect two preliminary estimates.

Lemma 1.5. Let $g^{x}$ and $g_{h}^{x}$ be defined as above, then

i) For any $M \geq 0$

$$
\left\|g^{x}-g_{h}^{x}\right\|_{W_{1}^{1}\left(B_{M h}(x)\right)} \leq C M^{N / 2} h .
$$

ii) If $d_{j} \geq M h \geq 8 k h$ and $\Omega_{j}$ is defined by (1.17), then

$$
\left\|g^{x}-g_{h}^{x}\right\|_{W_{1}^{1}\left(\Omega_{j}\right)} \leq C\left(\frac{h^{r-1}}{d_{j}^{r-2}}+d_{j}^{-1}\left\|g^{x}-g_{h}^{x}\right\|_{L_{1}\left(\Omega_{j}^{\prime}\right)}\right),
$$

where $C$ is independent of $g^{x}, g_{h}^{x}, x, h$ and $j$.

Proof. To prove (1.25) we use the Cauchy-Schwarz inequality, (1.16) and (1.20) to obtain

$$
\begin{aligned}
\left\|g^{x}-g_{h}^{x}\right\|_{W_{1}^{1}\left(B_{M h}(x)\right)} & \leq C(M) h^{N / 2}\left\|g^{x}-g_{h}^{x}\right\|_{W_{2}^{1}\left(B_{M h}(x)\right)} \\
& \leq C(M) h^{N / 2+1}\|\eta\|_{L_{2}\left(B_{2 k h}(x)\right)} \leq C M^{N / 2} h .
\end{aligned}
$$

To prove (1.26) we use the Cauchy-Schwarz inequality and (1.18) to obtain

$$
\begin{aligned}
& \left\|g^{x}-g_{h}^{x}\right\|_{W_{1}^{1}\left(\Omega_{j}\right)} \leq C d_{j}^{N / 2}\left\|g^{x}-g_{h}^{x}\right\|_{W_{2}^{1}\left(\Omega_{j}\right)} \\
& \quad \leq C\left(d_{j}^{N / 2} h^{r-1}\left\|g^{x}\right\|_{W_{2}^{r}\left(\Omega_{j}^{\prime}\right)}+d_{j}^{-1}\left\|g^{x}-g_{h}^{x}\right\|_{L_{1}\left(\Omega_{j}^{\prime}\right)}\right) .
\end{aligned}
$$

Since for $w \in B_{2 k h}$ and $y \in \Omega_{j}^{\prime},|w-y| \geq \frac{1}{2} d_{j}$, then in view of (1.8) it follows that for any $|\alpha| \leq r$

$$
\begin{aligned}
\left|D_{y}^{\alpha} g^{x}(y)\right| & \left.\leq \int_{B_{2 k h}(x)}|\eta(w)| \mid D_{y}^{\alpha} G^{y}(w)\right) \mid d w \\
& \leq \frac{C h^{N / 2}\|\eta\|_{L_{2}\left(B_{2 k h}(x)\right)}}{d_{j}^{N-2+r}} \leq \frac{C}{d_{j}^{N-2+r}},
\end{aligned}
$$

then

$$
\left\|g^{x}\right\|_{W_{2}^{r}\left(\Omega_{j}\right)} \leq \frac{C}{d_{j}^{N / 2-2+r}} .
$$

The inequality (1.26) follows from (1.27) and (1.28).

We shall need one more approximation result. For each $\lambda \in C_{0}^{\infty}\left(\Omega_{j}\right)$ with

$$
\|\lambda\|_{L_{\infty}\left(\Omega_{j}\right)}=1
$$

let $z$ be the solution of

$$
A(v, z)=(\lambda, v) \quad \text { for all } v \in W_{2}^{1}(\Omega) .
$$

Lemma 1.6. There exists a $\chi \in S_{r}^{h}(\Omega)$ such that

$$
\|z-\chi\|_{W_{\infty}^{1}\left(\Omega / \Omega_{j}^{\prime \prime}\right)} \leq C h^{r-1} d_{j}^{2-r}
$$

and

$$
\|z-\chi\|_{W_{2}^{1}\left(\Omega_{j}^{\prime \prime}\right)} \leq C h d_{j}^{N / 2},
$$

where in (1.31) and (1.32) $C$ is independent of $z, \lambda, h$ and $j$. 
Proof. In view of (1.9), there exists a $\chi \in S_{r}^{h}(\Omega)$ satisfying

$$
\|z-\chi\|_{W_{\infty}^{1}\left(\Omega / \Omega_{j}^{\prime \prime}\right)} \leq C h^{r-1}\|z\|_{W_{\infty}^{r}\left(\Omega / \Omega_{j}^{\prime}\right)} .
$$

If $w \in \Omega_{j}^{\prime}$ and $y \in \Omega_{j}, \frac{d_{j}}{2} \leq|w-y|$. Hence for any $|\alpha| \leq r$

$$
\left|D^{\alpha} z(y)\right| \leq \int_{\Omega_{j}}|\lambda(w)|\left|D_{y}^{\alpha} G^{y}(w)\right| d w \leq C\|\lambda\|_{L_{\infty}\left(\Omega_{j}\right)} d_{j}^{2-r} \leq C d_{j}^{2-r},
$$

where we have used (1.8) and (1.29). Together these last two estimates prove (1.31). Using (1.9), (1.7) and (1.29)

$$
\|z-\chi\|_{W_{2}^{1}\left(\Omega_{j}^{\prime \prime}\right)} \leq C h\|z\|_{W_{2}^{2}(\Omega)} \leq C h\|\lambda\|_{L_{2}\left(\Omega_{j}\right)} \leq C h d_{j}^{N / 2}\|\lambda\|_{L_{\infty}\left(\Omega_{j}\right)} \leq C h d_{j}^{N / 2},
$$

which proves (1.32).

We shall also be interested in pointwise error estimates for $\frac{\partial}{\partial x_{i}}\left(u-u_{h}\right)$. For this we shall need analogues of Lemmas 1.4 and 1.5. To begin with, using (1.10) and (1.11) and following a similar procedure as in deriving (1.22), we easily arrive at

$$
\begin{aligned}
\| \frac{\partial}{\partial x_{i}}\left(u-u_{h}\right) & \left\|_{L_{\infty}\left(B_{h}(x)\right)} \leq C\right\| \frac{\partial(u-\chi)}{\partial x_{i}} \|_{L_{\infty}\left(B_{K h}(x)\right)} \\
& +C h^{-N / 2-1}\left\|\frac{\partial}{\partial x_{i}}\left(u-u_{h}\right)\right\|_{W_{2}^{-1}\left(B_{K h}(x)\right)} .
\end{aligned}
$$

Now by duality

$$
\begin{aligned}
\left\|\frac{\partial}{\partial x_{i}}\left(u-u_{h}\right)\right\|_{W_{2}^{-1}\left(B_{K h}(x)\right)} & \sup =\operatorname{suc}_{\substack{\psi \in C_{0}^{\infty}\left(B_{K h}(x)\right) \\
\|\psi\|_{W_{2}^{1}\left(B_{K h}(x)\right)}=1}}\left(h^{-N / 2-1} \frac{\partial}{\partial x_{i}}\left(u-u_{h}\right), \psi\right) \\
= & \sup _{\substack{\psi \in C_{0}^{\infty}\left(B_{K h}(x)\right) \\
\|\psi\|_{W_{2}^{1}\left(B_{K h}(x)\right)}=1}}\left(u-u_{h},-h^{-N / 2-1} \frac{\partial \psi}{\partial x_{i}}\right) .
\end{aligned}
$$

Now for each such $\psi$ let $\widetilde{\eta}=h^{-N / 2-1} \psi$ and let $\widetilde{g}^{x}$ be the solution of

$$
A\left(v, \widetilde{g}^{x}\right)=\left(v, \frac{-\partial \widetilde{\eta}}{\partial x_{i}}\right) \quad \text { for all } v \in W_{2}^{1}(\Omega)
$$

Furthermore let $\widetilde{g}_{h}^{x} \in S_{r}^{h}$ be its finite element approximation satisfying

$$
A\left(\varphi, \widetilde{g}^{x}-\widetilde{g}_{h}^{x}\right)=0 \quad \text { for all } \varphi \in S_{r}^{h}(\Omega) .
$$

Using (1.34), (1.35) and (1.36)

$$
\begin{aligned}
\left(u-u_{h}, h^{-N / 2-1} \frac{\partial \widetilde{\eta}}{\partial x_{i}}\right) & =A\left(u-u_{h}, \widetilde{g}^{x}\right)=A\left(u-u_{h}, \widetilde{g}^{x}-\widetilde{g}_{h}^{x}\right) \\
& =A\left(u, \widetilde{g}^{x}-\widetilde{g}_{h}^{x}\right)=A\left(u-\chi, \widetilde{g}^{x}-\widetilde{x}_{h}^{x}\right) \\
& \leq\left\|\widetilde{g}^{x}-\widetilde{g}_{h}^{x}\right\|_{W_{1}^{1}(\Omega), x-s}\|u-\chi\|_{W_{\infty}^{1}(\Omega), x, s} .
\end{aligned}
$$

Combining this estimate with (1.34) and (1.33) we have proved the following: 
Lemma 1.7. Let $\widetilde{\eta}, \widetilde{g}^{x}$ and $\widetilde{g}_{h}^{x}$ be defined as above, then for any $1 \leq i \leq N$

$$
\begin{aligned}
& \left\|\frac{\partial}{\partial x_{i}}\left(u-u_{h}\right)\right\|_{L_{\infty}\left(B_{h}\left(x_{0}\right)\right)} \\
& \quad \leq C\left(1+\sup _{\widetilde{g}^{x}}\left\|\widetilde{g}^{x}-\widetilde{g}_{h}^{x}\right\|_{W_{1}^{1}(\Omega), x,-s}\right)\|u-\chi\|_{W_{\infty}^{1}(\Omega), x, s} .
\end{aligned}
$$

We now present the analogue of Lemma 1.5 for the function $\widetilde{g}^{x}-\widetilde{g}_{h}^{x}$.

Lemma 1.8. Let $\widetilde{g}^{x}$ and $\widetilde{g}_{h}^{x}$ be defined as above, then

i) For any $M \geq 0$

$$
\left\|\widetilde{g}^{x}-\widetilde{g}_{h}^{x}\right\|_{W_{1}^{1}\left(B_{M h}(x)\right)} \leq C M^{N / 2} .
$$

ii) If $d_{j} \geq M h \geq 9 K h$ and $\Omega_{j}$ is defined by (1.17), then

$$
\left\|\widetilde{g}^{x}-\widetilde{g}_{h}^{x}\right\|_{W_{1}^{1}\left(\Omega_{j}\right)} \leq C\left(\frac{h^{r-1}}{d_{j}^{r-1}}+d_{j}^{-1}\left\|\widetilde{g}^{x}-\widetilde{g}_{h}^{x}\right\|_{L_{1}\left(\Omega_{j}^{\prime}\right)}\right),
$$

where $C$ is independent of $\widetilde{g}^{x}, \widetilde{g}_{h}^{x}, x, h$ and $j$.

Proof. To prove (1.38), notice that using (1.16) and (1.35)

$$
\begin{aligned}
\left\|\widetilde{g}^{x}-\widetilde{g}_{h}^{x}\right\|_{W_{1}^{1}\left(B_{M h}(x)\right)} & \leq C M^{N / 2} h^{N / 2}\left\|\widetilde{g}-\widetilde{g}_{h}^{x}\right\|_{W_{2}^{1}\left(B_{M h}(x)\right)} \\
& \leq C M^{N / 2} h^{N / 2+1}\left\|\frac{\partial \widetilde{\eta}}{\partial x_{i}}\right\|_{L_{2}(\Omega)} \leq C M^{N / 2} .
\end{aligned}
$$

The proof of (1.39) is similar to the proof of (1.26)

$$
\begin{aligned}
& \left\|\widetilde{g}^{x}-\widetilde{g}_{h}^{x}\right\|_{W_{1}^{1}\left(\Omega_{j}\right)} \leq C d_{j}^{N / 2}\left\|\widetilde{g}^{x}-\widetilde{g}_{h}^{x}\right\|_{W_{2}^{1}\left(\Omega_{j}\right)} \\
& \quad \leq C\left(d_{j}^{N / 2} h^{r-1}\left\|\widetilde{g}^{x}\right\|_{W_{2}^{r}\left(\Omega_{j}^{\prime}\right)}+d_{j}^{-1}\left\|\widetilde{g}^{x}-\widetilde{g}_{h}^{x}\right\|_{L_{1}\left(\Omega_{j}\right)}\right) .
\end{aligned}
$$

Since for $w \in B_{2 K h}$ and $y \in \Omega_{j}^{\prime},|w-y| \geq \frac{1}{2} d_{j}$, then in view of (1.8) it follows that for any $|\alpha| \leq r$

$$
\begin{gathered}
D^{\alpha} \widetilde{g}^{x}(y)=\int_{B_{2 K h}}-\frac{\partial \widetilde{\eta}(w)}{\partial w_{i}} D_{y}^{\alpha} G^{y}(w) d w=\int_{B_{2 K h}} \widetilde{\eta}(w) \frac{\partial}{\partial w_{i}}\left(D_{y}^{\alpha} G^{y}(w)\right) d w \\
\left|D^{\alpha} \widetilde{g}^{x}(y)\right| \leq \frac{C h^{N / 2}\|\widetilde{\eta}\|_{L_{2}\left(B_{2 K h}(x)\right)}}{d_{j}^{N-1+r}} \leq \frac{C h^{N / 2+1}\|\widetilde{\eta}\|_{W_{2}^{1}\left(B_{2 K h}(x)\right)}}{d_{j}^{N-1+r}}
\end{gathered}
$$

and therefore

$$
\left\|\widetilde{g}^{x}\right\|_{W_{2}^{r}\left(\Omega_{j}\right)} \leq \frac{C}{d_{j}^{N / 2-1+r}}
$$

and (1.39) follows from this and (1.40).

\section{Pointwise estimates For $\left(u-u_{h}\right)(x)$}

(A) Statements of results. This section will be devoted to the derivation of pointwise estimates for $\left(u-u_{h}\right)(x)$ satisfying

$$
A\left(u-u_{h}, \varphi\right)=0 \text { for all } \varphi \in S_{r}^{h}(\Omega) .
$$

This will then be generalized to $u-u_{h}$ satisfying

$$
A\left(u-u_{h}, \varphi\right)=F(\varphi) \text { for all } \varphi \in S_{r}^{h}(\Omega),
$$


where $F(\varphi)$ is a bounded linear functional on $W_{1}^{1}(\Omega)$. Such equations often arise when considering problems leading to perturbations of the bilinear form $A$ (see, for example, Nitsche and Schatz [6], Schatz and Wahlbin [10], Schatz, Sloan and Wahlbin [12]).

The main result of this section is as follows:

Theorem 2.1. Suppose that A.1-A.4 are satisfied and $u \in W_{\infty}^{1}(\Omega)$ and $u_{h} \in$ $S_{r}^{h}(\Omega)$ satisfies (2.1). Let $x \in \bar{\Omega}$ and s satisfy $0 \leq s \leq r-2, r \geq 2$. Then there exists a constant $C$ independent of $x, u, u_{h}$ and $h$ such that

$$
\left|\left(u-u_{h}\right)(x)\right| \leq\left\|u-u_{h}\right\|_{L_{\infty}(\Omega), x, s} \leq C h\left(\ln \frac{1}{h}\right)^{\bar{s}} \inf _{\chi \in S_{r}^{h}}\|u-\chi\|_{W_{\infty}^{1}(\Omega), x, s} .
$$

Here $\bar{s}=0$ if $0 \leq s<r-2$ and $\bar{s}=1$ if $s=r-2$.

The generalization of Theorem 2.1 is as follows:

Theorem 2.2. Suppose the conditions of Theorem 2.1 are satisfied except that $u-u_{h}$ satisfies (2.2). Then

$$
\begin{aligned}
\left|\left(u-u_{h}\right)(x)\right| \leq C & \left(h\left(\ln \frac{1}{h}\right)^{\bar{s}} \inf _{\chi \in S_{r}^{h}}\|u-\chi\|_{W_{\infty}^{1}(\Omega), x, s}\right. \\
& \left.+h\left(\ln \frac{1}{h}\right)^{\bar{s}}\|||\|_{-1, x, s}+\left(\ln \frac{1}{h}\right) \||| F||_{-2}\right) .
\end{aligned}
$$

Here $s, \bar{s}$ and $r$ are as in Theorem 2.1 and $C$ is independent of $x, u, u_{h}, s, h$ and F. Furthermore

$$
\|||||_{-1, x, s}=\sup _{\substack{\varphi \in W_{1}^{1}(\Omega) \\\|\varphi\|_{W_{1}^{1}(\Omega), x,-s}=1}} F(\varphi)
$$

and

$$
|||F|||_{-2}=\sup _{\substack{\varphi \in W_{1}^{2}(\Omega) \\\|\varphi\|_{W_{1}^{2}(\Omega)}=1}} F(\varphi) .
$$

Remark. Suppose that in addition to A.1-A.4 the following assumption on the subspace holds:

$$
\bar{\Omega}=\bigcup_{j=1}^{N(h)} \bar{\tau}_{j}^{h},
$$

where the $\tau_{j}^{h}$ are disjoint sets having the property that there exists a constant $C$ such that for any $f \in W_{1}^{1}\left(\tau_{j}^{h}\right), 0<h<1, j=1, \ldots, N(h)$,

$$
\int_{\partial \tau_{j}^{h}}|f| d s \leq C\left\{h^{-1}\|f\|_{L_{1}\left(\tau_{j}^{h}\right.}+\|f\|_{W_{1}^{1}\left(\tau_{j}^{h}\right)}\right\} .
$$

Then using the technique introduced in [11] one can replace the term

$$
h \inf _{\chi \in S_{r}^{h}}\|u-\chi\|_{W_{1}^{1}(\Omega), x, s}
$$

in (2.3) and (2.4) with

$$
\inf _{\chi \in S_{r}^{h}}\|u-\chi\|_{L_{\infty}(\Omega), x, s}
$$


(B) Proof of Theorem 2.1. As remarked in the introduction the proof of (2.3) has much in common with previous proofs of $L_{\infty}$ estimates. With some essential modifications, the proof given here will follow in outline that given in Schatz and Wahlbin [9], [10].

In view of Lemma 1.4, we have for any $\chi \in S_{r}^{h}(\Omega)$

$$
\left|\left(u-u_{h}\right)(x)\right| \leq C\left(\left\|g^{x}-g_{h}^{x}\right\|_{W_{1}^{1}(\Omega), x,-s}+h\right)\|u-\chi\|_{W_{\infty}^{1}(\Omega), x, s},
$$

where $g^{x}(y)$ satisfies (1.20) and $g_{h}^{x}(y)$ satisfies (1.21). The main step in the proof of $(2.3)$ is the following:

Lemma 2.1. Under the conditions of Theorem 2.1

$$
\left\|g^{x}-g_{h}^{x}\right\|_{W_{1}^{1}(\Omega), x,-s} \leq C h\left(\ln \frac{1}{h}\right)^{\bar{s}} .
$$

Assuming (2.8) for the moment, let us complete the proof of Theorem 2.1. In fact combining (2.8) with (2.7) it follows that for any $x \in \bar{\Omega}$ and $\chi \in S_{r}^{h}(\Omega)$

$$
|(u-u)(x)| \leq C h\left(\ln \frac{1}{h}\right)^{\bar{s}}\|u-\chi\|_{W_{\infty}^{1}(\Omega), x, s}
$$

where $C$ is as in Theorem 2.1. The inequality (2.3) follows from (2.9) and a simple inequality. To see this first notice that for any $x, y$ and $w \in \mathbb{R}^{N}$

$$
\begin{aligned}
& \left(\frac{h}{|y-x|+h}\right)\left(\frac{h}{|y-w|+h}\right) \\
& \quad \leq\left(\frac{h^{2}}{|x-w|+h}\right)\left(\frac{(|x-y|+h)+(|y-w|+h)}{(|x-y|+h)(|y-w|+h)}\right) \\
& \quad \leq \frac{2 h}{|x-w|+h} .
\end{aligned}
$$

Applying (2.9) with $x$ replaced by $y$, then multiplying both sides by $\left(\frac{h}{(|x-y|+h)}\right)^{s}$ and using (2.10)

$$
\begin{aligned}
& \left|\left(\frac{h}{|x-y|+h}\right)^{s}\left(u-u_{h}\right)(y)\right| \\
& \leq C h\left(\ln \frac{1}{n}\right)^{s}\left(\left\|\left(\frac{h}{|x-y|+h}\right)^{s}\left(\frac{h}{|y-w|+h}\right)^{s}(u-\chi)(w)\right\|_{L_{\infty}(\Omega)}\right. \\
& \left.\quad+\left\|\left(\frac{h}{|x-y|+h}\right)^{s}\left(\frac{h}{|y-w|+h}\right)^{s} \nabla(u-\chi)(w)\right\|_{L_{\infty}(\Omega)}\right) \\
& \leq 2^{s} C h\left(\ln \frac{1}{h}\right)^{s}\left(\left\|\left(\frac{h}{|x-w|+h}\right)^{s}(u-\chi)(w)\right\|_{L_{\infty}(\Omega)}\right. \\
& \left.\quad+\left\|\left(\frac{h}{|x-w|+h}\right)^{s} \nabla(u-\chi)(w)\right\|_{L_{\infty}(\Omega)}\right) .
\end{aligned}
$$

Taking the supremum over $y \in \bar{\Omega}$

$$
\left\|u-u_{h}\right\|_{L_{\infty}(\Omega), x, s} \leq C h\left(\ln \frac{1}{h}\right)^{\bar{s}}\left(\|u-\chi\|_{W_{\infty}^{1}(\Omega), x, s}\right),
$$

which completes the proof of (2.3) provided (2.8) holds. 
We now turn to the proof of (2.8). Let $M \geq 8 k>8$ ( $k$ defined in Section 1$)$ be a constant which will be chosen later on to be sufficiently large. For convenience we shall choose $M$ to begin with so that for some integer $J$

$$
M h=2^{-J} .
$$

Notice that since $M>1$

$$
J=\ln _{2} \frac{1}{M}+\ln _{2} \frac{1}{h} \leq \ln _{2} \frac{1}{h}
$$

Set $E \equiv g^{x}-g_{h}^{x}$, then

$$
\|E\|_{W_{1}^{1}(\Omega), x,-s} \leq C M^{s}\|E\|_{W_{1}^{1}\left(B_{M h}(x)\right)}+2^{s} \sum_{j=0}^{J} \frac{d_{j}^{s}}{h^{s}}\|E\|_{W_{1}^{1}\left(\Omega_{j}\right)},
$$

where we have assumed without loss of generality that $\operatorname{diam}(\Omega) \leq 1$. Using $(1.25)$, (1.26) and the fact that $M \geq 8 k \geq 8$

$$
\begin{aligned}
& \|E\|_{W_{1}^{1}(\Omega), x,-s} \\
& \leq C M^{N / 2+s} h+C\left(\sum_{j=0}^{J} h\left(\frac{h}{d_{j}}\right)^{r-2-s}+\sum_{j=0}^{J} \frac{d_{j}^{-1+s}}{h^{s}}\|E\|_{L_{1}\left(\Omega_{j}^{\prime}\right)}\right) \\
& \leq C_{1}\left(M^{N / 2+s} h+h \delta(s)+h^{-1}\|E\|_{L_{1}(\Omega), x,-s+1}\right),
\end{aligned}
$$

where $C_{1}$ is independent of $h, M, s$ and $x$. Here we have used the fact that since $d_{j}=2^{-j}$ and $J \leq \ln \frac{1}{h}$ and $\gamma=r-2-s$,

$$
\sum_{j=1}^{J}\left(\frac{h}{d_{j}}\right)^{\gamma} \leq \begin{cases}\ln \frac{1}{h} & \text { if } \gamma=0 \\ \frac{1}{M^{\gamma}}\left(\frac{1-\left(\frac{1}{2}\right)^{\gamma \ln \frac{1}{h}}}{1-\left(\frac{1}{2}\right)^{\gamma}}\right) & \text { if } 0<\gamma \leq r-2 .\end{cases}
$$

We shall now estimate the last term on the right in (2.14).

$$
h^{-1}\|E\|_{L_{1}(\Omega), x,-s+1} \leq C M^{s} h^{-1}\|E\|_{L_{1}\left(B_{M h}(x)\right)}+2^{s} \sum_{j=0}^{J} \frac{d_{j}^{-1+s}}{h^{s}}\|E\|_{L_{1}\left(\Omega_{j}\right)} .
$$

Using (1.16) and (1.20)

$$
\begin{aligned}
C M^{s} h^{-1}\|E\|_{L_{1}\left(B_{M h}(x)\right)} & \leq C M^{N / 2+s} h^{N / 2-1}\|E\|_{L_{2}\left(B_{M h}(x)\right)} \\
& \leq C M^{N / 2+s} h^{N / 2+1}\|\eta\|_{L_{2}\left(B_{k h}(x)\right)} \\
& \leq C M^{N / 2+s} h .
\end{aligned}
$$

Furthermore for each $0 \leq j \leq J$, it follows that

$$
\|E\|_{L_{1}\left(\Omega_{j}\right)}=\sup _{\substack{\lambda \in C_{0}^{\infty}\left(\Omega_{j}\right) \\\|\lambda\|_{L_{\infty}\left(\Omega_{j}\right)}=1}}(E, \lambda) .
$$

For each $\lambda$, let $z$ be the solution of

$$
A(z, v)=(\lambda, v) \text { for all } v \in W_{2}^{1}(\Omega) .
$$

Then for any $\chi \in S_{r}^{h}(\Omega)$ it follows that

$$
(E, \lambda)=A(z, E)=A(z-\chi, E) \text {. }
$$


Hence

$$
\begin{aligned}
|(E, \lambda)| \leq C\|E\|_{W_{1}^{1}\left(\Omega / \Omega_{j}^{\prime \prime}\right)}\|z-\chi\|_{W_{\infty}^{1}\left(\Omega / \Omega_{j}^{\prime \prime}\right)} & \\
& +C\|E\|_{W_{2}^{1}\left(\Omega_{j}^{\prime \prime}\right)}\|z-\chi\|_{W_{2}^{1}\left(\Omega_{j}^{\prime \prime}\right)} \\
= & I_{1}+I_{2} .
\end{aligned}
$$

In view of (1.31)

$$
I_{1} \leq C \frac{h^{r-1}}{d_{j}^{r-2}}\|E\|_{W_{1}^{1}(\Omega), x,-s}
$$

and from (1.26) and (1.32)

$$
I_{2} \leq C \frac{h^{r}}{d_{j}^{r-2}}+C \frac{h}{d_{j}}\|E\|_{L_{1}\left(\Omega_{j}^{\prime \prime \prime}\right)}
$$

Collecting these estimates into (2.18) we obtain

$$
\|E\|_{L_{1}\left(\Omega_{j}\right)} \leq C\left(\frac{h^{r}}{d_{j}^{r-2}}+\frac{h^{r-1}}{d_{j}^{r-2}}\|E\|_{W_{1}^{1}(\Omega), x,-s}+\frac{h^{s+1}}{d_{j}^{s}} h^{-1}\|E\|_{L_{1}(\Omega), x,-s+1}\right),
$$

where $C$ is independent of $x, h, M$ and $j$.

Multiplying both sides by $d_{j}^{s-1} / h^{s}$, summing for $0 \leq j \leq J$ and then using the result together with (2.17) in (2.16) we obtain

$$
\begin{aligned}
h^{-1}\|E\|_{L_{1}(\Omega), x,-s+1} \leq & C M^{N / 2+s} h+C h\left(\sum_{j=0}^{J}\left(\frac{h}{d_{j}}\right)^{r-1-s}\right) \\
& +C\left(\sum_{j=0}^{J}\left(\frac{h}{d_{j}}\right)^{r-1-s}\right)\|E\|_{W_{1}^{1}(\Omega), x,-s} \\
& +C\left(\sum_{j=0}^{J}\left(\frac{h}{d_{j}}\right)\right) h^{-1}\|E\|_{L_{1}(\Omega), x,-s+1} .
\end{aligned}
$$

Since $r-1-s \geq 1$, from (2.15) and (2.16) we obtain

$$
\begin{aligned}
& h^{-1}\|E\|_{L_{1}(\Omega), x,-s+1} \leq C M^{N / 2+s} h+\frac{C_{2}}{M}\|E\|_{W_{1}^{1}(\Omega), x,-s} \\
&+\frac{C_{2}}{M} h^{-1}\|E\|_{L_{1}(\Omega), x,-s+1},
\end{aligned}
$$

where $C_{2}$ is independent of $x, M, s$ and $h$. Choosing $M$ sufficiently large so that $\frac{C_{2}}{M} \leq \frac{1}{2}$ we easily find

$$
h^{-1}\|E\|_{L_{1}(\Omega), x,-s+1} \leq 2 C_{2} M^{N / 2+s} h+\frac{2 C_{2}}{M}\|E\|_{W_{1}^{1}(\Omega), x,-s} .
$$

The inequality (2.8) follows upon substituting (2.23) into (2.14)

$$
\|E\|_{W_{1}^{1}(\Omega), x,-s} \leq C M^{N / 2+s} h+C h\left(\ln \frac{1}{h}\right)^{\bar{s}}+\frac{2 C_{1} C_{2}}{M}\|E\|_{W_{1}^{1}(\Omega), x,-s}
$$

and choosing $M$ sufficiently large so that $\frac{2 C_{1} C_{2}}{M} \leq \frac{1}{2}$, which completes the proof. 
(C) Proof of Theorem 2.2. The proof of (2.4) is almost exactly the same as that of (2.3) with one difference. In fact because of (2.2), instead of (2.14) we have

$$
\left|\left(u-u_{h}\right)(x)\right| \leq C\left(\left\|g^{x}-g_{h}^{x}\right\|_{W_{1}^{1}(\Omega), x,-s}+h\right)\|u\|_{W_{\infty}^{1}(\Omega), x, s}+\left|F\left(g_{h}^{x}\right)\right| .
$$

Now in view of $(2.8)$

$$
\begin{aligned}
\left|F\left(g_{h}^{x}\right)\right| & \leq\left|F\left(g^{x}-g_{h}^{x}\right)\right|+\left|F\left(g^{x}\right)\right| \\
& \leq|||F|\left\|\left.\right|_{-1, x, s}\right\| g^{x}-g_{h}^{x}\left\|_{W_{1}^{1}(\Omega), x, s}+\right\||| F||_{-2}\left\|g^{x}\right\|_{W_{1}^{2}(\Omega)} \\
& \leq C h\left(\ln \frac{1}{h}\right)^{\bar{s}}|||F|\left\|_{-1, x, s}+\ln \frac{1}{h}|||F|\right\|_{-2},
\end{aligned}
$$

where we have used the fact that

$$
\begin{aligned}
\left\|g^{x}\right\|_{W_{1}^{2}(\Omega)} & \leq C M^{N / 2} h^{N / 2}\left\|g^{x}\right\|_{W_{2}^{2}\left(B_{M h}(x)\right)}+C \sum_{j=0}^{J} d_{j}^{N / 2}\left\|g^{x}\right\|_{W_{2}^{2}\left(\Omega_{j}\right)} \\
& \leq C M^{N / 2}+C \sum_{j=0}^{J} 1 \\
& \leq C J \leq C\left(\ln \frac{1}{h}\right) .
\end{aligned}
$$

Together, (2.24) and (2.25) imply (2.4) which completes the proof.

\section{ESTIMATES FOR FIRST DERIVATIVES}

(A) Statement of results. Here we shall be concerned with weighted $W_{\infty}^{1}$ estimates for $u-u_{h}$. The main result of this section, Theorem 3.1, is the analogue for first derivatives of Theorem 2.1.

Theorem 3.1. Suppose that A.1-A.4 are satisfied and that $u \in W_{\infty}^{1}(\Omega)$ and $u_{h} \in$ $S_{r}^{h}(\Omega)$ satisfy (2.1). Let $x \in \bar{\Omega}$ and s satisfy $0 \leq s \leq r-1$, where $r \geq 2$. Then there exists a constant $C$ independent of $x, u, u_{h}$ and $h$ such that

$$
\left\|u-u_{h}\right\|_{W_{\infty}^{1}\left(B_{h}(x)\right)} \leq 2\left\|u-u_{h}\right\|_{W_{\infty}^{1}(\Omega), x, s} \leq C\left(\ln \frac{1}{h}\right)^{\overline{\bar{s}}} \inf _{\chi \in S_{r}^{h}}\|u-\chi\|_{W_{\infty}^{1}(\Omega), x, s} .
$$

Here $\overline{\bar{s}}=0$ if $0 \leq s<r-1$ and $\overline{\bar{s}}=1$ if $s=r-1$.

Remark 3.1. Notice that the range of $s$ is greater in Theorem 3.1 than in Theorem 2.1. Here $s$ may be chosen $s>0$ for any $r \geq 2$.

Theorem 3.2. Suppose that the conditions of Theorem 3.1 hold where now (2.2) is satisfied. Then,

$$
\begin{aligned}
& \left\|u-u_{h}\right\|_{W_{\infty}^{1}\left(B_{h}(x)\right)} \leq 2\left\|u-u_{h}\right\|_{W_{\infty}^{1}(\Omega), x, s} \\
& \quad \leq C\left(\inf _{\chi \in S_{r}^{h}}\|u-\chi\|_{W_{\infty}^{1}(\Omega), x, s}+\ln \left(\frac{1}{h}\right)\||| F \mid\|_{-1}\right),
\end{aligned}
$$

where

$$
\|||||_{-1}=\sup _{\substack{\varphi \in W_{1}^{1}(\Omega) \\\|\varphi\|_{W_{1}^{1}(\Omega)}=1}}|F(\varphi)| .
$$


Proof of Theorem 3.1. Let us first remark that the inequality

$$
\left\|u-u_{h}\right\|_{W_{\infty}^{1}(\Omega), x, s} \leq C \inf _{\chi \in S_{r}^{h}}\|u-\chi\|_{W_{\infty}^{1}(\Omega), x, s}
$$

follows, using (2.10), from the inequality

$$
\left\|u-u_{h}\right\|_{W_{\infty}^{1}\left(B_{h}(x)\right)} \leq C \inf _{\chi \in S_{r}^{h}}\|u-\chi\|_{W_{\infty}^{1}(\Omega), x, s} .
$$

Furthermore it follows from Theorem 2.1 that for $0 \leq s<r-1$

$$
\left\|u-u_{h}\right\|_{L_{\infty}\left(B_{h}(x)\right)} \leq C \inf \|u-\chi\|_{W_{\infty}^{1}(\Omega), x, s}
$$

Hence Theorem 3.1 will follow once we have proved that for any $1 \leq i \leq N$

$$
\left\|\frac{\partial}{\partial x_{i}}\left(u-u_{h}\right)\right\|_{L_{\infty}\left(B_{h}(x)\right)} \leq C \inf _{\chi \in S_{r}^{h}}\|u-\chi\|_{W_{\infty}^{1}(\Omega), x, s} .
$$

On the other hand, it follows from Lemma 1.7 that

$$
\begin{aligned}
\| \frac{\partial}{\partial x_{i}} & \left(u-u_{h}\right) \|_{L_{\infty}\left(B_{h}(x)\right)} \\
& =C\left(1+\sup _{\psi}\left\|\widetilde{g}^{x}-\widetilde{g}_{h}^{x}\right\|_{W_{1}^{1}(\Omega), x,-s}\right) \inf _{\chi \in S_{r}^{h}}\|u-\chi\|_{W_{\infty}^{1}(\Omega), x, s}
\end{aligned}
$$

for all $\psi \in C_{0}^{\infty}\left(B_{k h}(x)\right)$ with $\|\psi\|_{W_{2}^{1}\left(B_{k h}(x)\right)}=1$. Here $\widetilde{g}^{x}$ satisfies (1.35), i.e.,

$$
A\left(v, \widetilde{g}^{x}\right)=\left(v,-h^{-N / 2} \frac{\partial \psi}{\partial x_{i}}\right) \quad \text { for all } v \in W_{2}^{1}(\Omega)
$$

and $\widetilde{g}_{h}^{x}$ satisfies $(1.36)$, i.e.,

$$
A\left(\varphi, \widetilde{g}^{x}-\widetilde{g}_{h}^{x}\right)=0 \quad \text { for all } \varphi \in S_{r}^{h}(\Omega) .
$$

Thus Theorem 3.1 will follow from (3.6) once we have proved the following analogue of Lemma 2.1:

Lemma 3.1. For any $\psi$ as above, let $\widetilde{g}^{x}$ and $\widetilde{g}_{h}^{x}$ satisfy (3.7) and (3.8). Then for $0 \leq s<r-1$

$$
\left\|\widetilde{g}^{x}-\widetilde{g}_{h}^{x}\right\|_{W_{1}^{1}(\Omega), x,-s} \leq C\left(\ln \frac{1}{h}\right)^{\overline{\bar{s}}},
$$

where $\overline{\bar{s}}=0$ if $0 \leq s<r-1, \bar{s}=1$ and $C$ is independent of $\psi, h$ and $x$.

Proof. The proof of (3.9) follows closely the proof of (2.8), the only difference being the use of Lemma 1.8 instead of Lemma 1.5. Hence we shall only indicate the differences. We shall start with the case that $0 \leq s<r-1$.

Set $\widetilde{E}=\widetilde{g}^{x}-\widetilde{g}_{h}^{x}$, then analogous to $(2.13)$ we have for $2^{-J}=M h$

$$
\|\widetilde{E}\|_{W_{1}^{1}(\Omega), x,-s} \leq C M^{s}\|\widetilde{E}\|_{W_{1}^{1}\left(B_{M h}(x)\right)}+2^{s} \sum_{j=0}^{J} \frac{d_{j}^{s}}{h^{s}}\|\widetilde{E}\|_{W_{1}^{1}\left(\Omega_{j}\right)} .
$$

In view of (1.38), (1.39) and (2.15)

$$
\|\widetilde{E}\|_{W_{1}^{1}(\Omega), x,-s} \leq C_{1}\left(M^{N / 2+s}+\delta(s)+h^{-1}\|\widetilde{E}\|_{L_{1}(\Omega), x,-s+1}\right)
$$

where $C_{1}$ is independent of $h, M$ and $x$.

The procedure for estimating $h^{-1}\|\widetilde{E}\|_{L_{1}(\Omega), x,-s+1}$ in this case is the same as that used for estimating $h^{-1}\|E\|_{L_{1}(\Omega), x,-s+1}$ in Theorem 2.1, where we now use (1.38) in 
place of (1.25) and (1.39) in place of (1.26). It is then easy to see that the estimates analogous to $(2.21)$ and $(2.22)$ are

$$
\begin{aligned}
& h^{-1}\|\widetilde{E}\|_{L_{1}(\Omega), x,-s+1} \\
& \leq C M^{N / 2+s}+C \sum_{j=0}^{J}\left(\frac{h}{d_{j}}\right)^{r-1-s}+C\left(\sum_{j=0}^{J}\left(\frac{h}{d_{j}}\right)^{r-1-s}\right)\|\widetilde{E}\|_{W_{1}^{1}(\Omega), x,-s} \\
& \quad+C\left(\sum_{j=0}^{J}\left(\frac{h}{d_{j}}\right)\right) h^{-1}\|\widetilde{E}\|_{L_{1}(\Omega), x,-s} \\
& \leq C M^{N / 2+s}+\frac{C_{2}}{M^{r-1-s}}\|\widetilde{E}\|_{W_{1}^{1}(\Omega), x,-s}+\frac{C_{2}}{M} h^{-1}\|\widetilde{E}\|_{L_{1}(\Omega), x,-s+1},
\end{aligned}
$$

where $C_{2}$ is independent of $x, M$ and $h$. Choosing $M$ sufficiently large so that $\frac{C_{2}}{M} \leq \frac{1}{2}$ in (3.12) and then combining the resulting inequality with (3.11) we arrive

$$
\|\widetilde{E}\|_{W_{1}^{1}(\Omega), x,-s} \leq C M^{N / 2}+\frac{2 C_{1} C_{2}}{M^{r-1-s}}\|\widetilde{E}\|_{W_{1}^{1}(\Omega), x-s} .
$$

For $r-1-s=\gamma>0$ we may further choose $M$ sufficiently large so that $\frac{2 C_{1} C_{2}}{M^{\gamma}} \leq \frac{1}{2}$, which completes the proof for this case.

We now turn to a proof of (3.9) in the case that $s=r-1$. We first note that the inequality (1.39) may be replaced by

$$
\|\widetilde{E}\|_{W_{1}^{1}\left(\Omega_{j}\right)} \leq C\left(\frac{h^{r-1}}{d_{j}^{r-1}}+d_{j}^{1-r}\|\widetilde{E}\|_{W_{1}^{2-r}\left(\Omega_{j}^{\prime}\right)}\right) .
$$

This is easily obtained by using (1.18) with $t=r-2$ in (1.40). Using (3.13) in (3.10) with $s=r-1$ we obtain instead of (3.11)

$$
\|\widetilde{E}\|_{W_{1}^{1}(\Omega), x, 1-r} \leq C\left(M^{N / 2+s}+\ln \frac{1}{h}+h^{1-r}\|\widetilde{E}\|_{W_{1}^{2-r}(\Omega)}\right) .
$$

Notice that the last norm on the right is not weighted. It will be estimated with a duality argument

$$
\|\widetilde{E}\|_{W_{1}^{2-r}(\Omega)}=\sup _{\substack{\psi \in C_{0}^{\infty}(\Omega) \\\|\psi\|_{W_{\infty}^{r-2}(\Omega)}=1}}(\widetilde{E}, \psi) .
$$

For each $\psi$, let $\Psi \in W_{p}^{r}(\Omega)$ for $2 \leq p<\infty$ satisfy

$$
A(\Psi, v)=(\psi, v) \quad \text { for all } v \in W_{2}^{1}(\Omega) .
$$

Then for each such $\psi$ and any $\chi \in S_{r}^{h}(\Omega)$

$$
(\widetilde{E}, \psi)=A(\Psi, \widetilde{E})=A(\Psi-\chi, \widetilde{E}) \leq C\|\widetilde{E}\|_{W_{1}^{1}(\Omega)}\|\Psi-\chi\|_{W_{\infty}^{1}(\Omega)} .
$$

Now from the case $s=0$ proved above

$$
\|\widetilde{E}\|_{W_{1}^{1}(\Omega)} \leq C
$$

and from (1.9b) and (1.7) and for the choice $p=\ln \frac{1}{h}$

$$
\begin{aligned}
\|\Psi-\chi\|_{W_{\infty}^{1}(\Omega)} & \leq C h^{r-1-N / p}\|\Psi\|_{W_{p}^{r}(\Omega)} \leq C p h^{r-1-N / p}\|\psi\|_{W_{p}^{r-2}(\Omega)} \\
& \leq C p h^{r-1-N / p} \leq C h^{r-1} \ln \frac{1}{h} .
\end{aligned}
$$


Using (3.18) and (3.19) in (3.17) and this in turn in (3.15) we obtain

$$
\|\widetilde{E}\|_{W_{1}^{2-r}(\Omega)} \leq C h^{r-1} \ln \frac{1}{h} .
$$

The case $s=r-1$ now follows by using (3.20) in (3.14). This completes the proof of Lemma 3.1.

(C) Proof of Theorem 3.2. The proof of (3.2) is almost exactly the same as that of (3.1) with one difference. This time instead of (3.6) we have

$$
\begin{aligned}
& \left\|\frac{\partial}{\partial x_{i}}\left(u-u_{h}\right)\right\|_{L_{\infty}\left(B_{h}(x)\right)} \\
& \quad \leq C\left(1+\sup _{\psi}\left\|\widetilde{g}^{x}-\widetilde{g}_{h}^{x}\right\|_{W_{1}^{1}(\Omega), x,-s}\right) \inf _{\chi \in S_{r}^{h}}\|u-\chi\|_{W_{\infty}^{1}(\Omega), x, s}+\sup _{\psi}\left|F\left(\widetilde{g}_{h}^{x}\right)\right|
\end{aligned}
$$

for all $\psi \in C_{0}^{\infty}\left(B_{k h}(x)\right),\|\psi\|_{W_{2}^{1}\left(B_{k h}(x)\right)}=1$.

Now

$$
\left|F\left(\widetilde{g}_{h}^{x}\right)\right| \leq|||F|\left\|\left.\right|_{-1}\right\| \widetilde{g}_{h}^{x}\left\|_{W_{1}^{1}(\Omega)} \leq\right\||| F|\||_{-1}\left(C+\left\|\widetilde{g}^{x}\right\|_{W_{1}^{1}(\Omega)}\right) .
$$

Furthermore using estimates of the type (1.41)

$$
\left\|\widetilde{g}^{x}\right\|_{W_{1}^{1}(\Omega)} \leq C\left(\ln \frac{1}{h}\right) .
$$

And (3.2) easily follows from these estimates and Lemma 3.1 .

\section{ERror EXPANSION INEQUALITIES}

(A) Preliminaries. Here we shall discuss some simple but useful consequences of Theorems 2.1 and 3.1. In particular estimates will be derived which for want of a better name we shall call "error expansion inequalities". These are bounds for the error at a point in terms of a sum of powers of $h$ multiplied by appropriate derivatives of $u$ taken at the point or a sufficiently close point. These expansions are a consequence of the weighted norm estimates and the fact that there are no polution effects in the smooth problems we are considering. In Part II of this work we shall show how localized versions of Theorems 2.1 and 3.1 lead to error expansion inequalities which may be applied to a variety of problems with both smooth and nonsmooth solutions (in which polution effects are present) to derive new superconvergence and extrapolation results.

For simplicity, it will be convenient for us to assume a strengthened form of A.1 in the case $p=\infty$.

A.5. Assume that the function $\chi \in S_{r}^{h}$ in A.1 satisfies

$$
\|u-\chi\|_{W_{\infty}^{1}\left(D_{1}\right)} \leq C h^{r-1}|u|_{W_{\infty}^{r}\left(D_{2}\right)}
$$

where $|\cdot|_{W_{\infty}^{r}(D)}$ denotes the semi-norm

$$
|u|_{W_{\infty}^{r}(D)}=\sum_{|\alpha|=r}\left\|D^{\alpha} u\right\|_{L_{\infty}(D)} .
$$

Consider now the weighted semi-norm

$$
|u|_{W_{\infty}^{r}(\Omega), x, s}=\sum_{|\alpha|=r}\left\|D^{\alpha} u\right\|_{L_{\infty}(\Omega), x, s}
$$


Then a simple consequence of the approximation assumption (4.1) is the fact that for any real $s$

$$
\|u-\chi\|_{W_{\infty}^{1}(\Omega), x, s} \leq C h^{r-1}|u|_{W_{\infty}^{r}(\Omega), x, s} .
$$

(B) The main results. Our first expansion inequality is as follows:

Theorem 4.1. Suppose the conditions of Theorem 2.1 are satisfied and in addition A.5 holds. Let $u \in W_{\infty}^{t}(\Omega)$ where $t$ is an integer $r+1 \leq t \leq 2 r-2$. Let $\widehat{C}>0$ be a fixed but arbitrary constant. For any $x \in \bar{\Omega}$, let $\widehat{x} \in \bar{\Omega}$ be an arbitrary point satisfying $|x-\widehat{x}| \leq \widehat{C} h$, then

$$
\begin{aligned}
& \left|\left(u-u_{h}\right)(x)\right| \leq C\left(\ln \frac{1}{h}\right)^{\bar{t}}\left(h^{r} \sum_{|\alpha|=r}\left|D^{\alpha} u(\widehat{x})\right|+\cdots\right. \\
& \left.+h^{t-1} \sum_{|\alpha|=t-1}\left|D^{\alpha} u(\widehat{x})\right|+h^{t}\|u\|_{W_{\infty}^{t}(\Omega)}\right) .
\end{aligned}
$$

Here $C$ is independent of $u, u_{h}, h, x$ and $\widehat{x} . \bar{t}=0$ if $t<2 r-2$ and $\bar{t}=1$ if $t=2 r-2$.

Before proving the error expansion inequality (4.5) let us make a few remarks.

Remark 4.1. The inequality (4.5) may be trivially converted to an equality of the form

$$
\begin{aligned}
\left|\left(u-u_{h}\right)(x)\right|=C & \left(x, \widehat{x}, h, u, u_{h}\right)\left(\ln \frac{1}{h}\right)^{\bar{t}}\left(h^{r} \sum_{|\alpha|=r}\left|D^{\alpha} u(\widehat{x})\right|+\cdots\right. \\
& \left.+h^{t-1} \sum_{|\alpha|=t-1}\left|D^{\alpha} u(\widehat{x})\right|+h^{t}\|u\|_{W_{\infty}^{t}(\Omega)}\right) .
\end{aligned}
$$

Here $C$ is a function of $x, \widehat{x}, h, u$, and $u_{h}$ but by (4.5) may be bounded by a constant which is independent of these quantities.

Remark 4.2. As discussed in the introduction (4.5) indicates a more local dependence of error on $u$ than indicated by (0.5). We hope to discuss the implications of this with respect to a posteriori estimates in another paper.

Remark 4.3. Higher order convergence than $h^{r}$ is obtained if appropriate derivatives of $u$ vanish at some point $\widehat{x}$. More precisely we have the following immediate consequence of (4.5).

Corollary 4.1. Suppose that the conditions of Theorem 4.1 are satisfied and in addition for some point $\widehat{x}$ with $|x-\widehat{x}| \leq \widehat{C} h, D^{\alpha} u(\widehat{x})=0$ for $r=|\alpha| \leq t-1$; then

$$
\left|\left(u-u_{h}\right)(x)\right| \leq\left(\ln \frac{1}{h}\right)^{\bar{t}} h^{t}|u|_{W_{t}^{r}(\Omega)} .
$$

Notice that under these conditions the maximum rate of convergence possible from (4.5) is $h^{2 r-2}\left(\ln \frac{1}{h}\right)$.

Remark 4.4. If $u$ belongs to the Hölder space $C^{\ell+\gamma}$, where $r \leq \ell \leq 2 r-3$ is an integer and $0<\gamma \leq 1$, then one can easily derive the expansion inequality

$$
\begin{aligned}
& \left|\left(u-u_{h}\right)(x)\right| \\
& \leq C\left(\ln \frac{1}{h}\right)^{\bar{\ell}}\left(h^{r} \sum_{|\alpha|=r}\left|D^{\alpha} u(\widehat{x})\right|+\cdots+h^{\ell} \sum_{|\alpha|=\ell}\left|D^{\ell} u(\widehat{x})\right|+h^{\ell+\gamma}\|u\|_{C^{\ell+\gamma}(\Omega)}\right) .
\end{aligned}
$$


Here $\bar{\ell}=0$ if $\ell+\gamma<2 r-2$ and $\bar{\ell}=1$ if $\ell+\gamma=2 r-2$.

Proof of Theorem 4.1. In view of (2.3) and (4.4) we immediately have for the choice $s=t-r$

$$
\left|\left(u-u_{h}\right)(x)\right| \leq C h^{r}\left(\ln \frac{1}{h}\right)^{\bar{t}}|u|_{W_{\infty}^{r}(\Omega), x, t-r} .
$$

Now for any multi-index $\alpha^{\prime}$ with $\left|\alpha^{\prime}\right|=r$ and any $y \in \Omega$

$$
\left|D^{\alpha^{\prime}} u(y)\right| \leq C\left(\sum_{0 \leq|\beta| \leq t-r-1}\left|D^{\alpha^{\prime}+\beta} u(\widehat{x})\right||y-\widehat{x}|^{|\beta|}+\|u\|_{W_{\infty}^{t}(\Omega)}|y-\widehat{x}|^{t-r}\right) .
$$

This follows trivially from Taylor's theorem when $\Omega$ is convex and in the nonconvex case by first extending $u$ continuously in $W_{\infty}^{t}$ to a ball containing $\Omega$. Now from $(4.9)$

$$
\begin{array}{r}
\left|D^{\alpha^{\prime}} u(y)\right| \leq C\left(\sum_{r \leq|\alpha| \leq t-1}\left|D^{\alpha} u(\widehat{x})\right|(|y-x|+|x-\widehat{x}|)^{|\alpha|-r}\right. \\
\left.\quad+\|u\|_{W_{\infty}^{t}(\Omega)}(|y-x|+|x-\widehat{x}|)^{t-1}\right) \\
\leq C\left(\sum_{r \leq|\alpha| \leq t-1}\left|D^{\alpha} u(\widehat{x})\right|(|y-x|+h)^{|\alpha|-r}\right. \\
\left.+\|u\|_{W_{\infty}^{t}(\Omega)}(|y-x|+h)^{t-r}\right) .
\end{array}
$$

Hence

$$
\frac{h^{t-r}}{(|y-x|+h)^{t-r}}\left|D^{\alpha^{\prime}} u(y)\right| \leq C \sum_{r \leq|\alpha| \leq t-1} h^{|\alpha|-r}\left|D^{\alpha} u(\widehat{x})\right|+h^{t-r}\|u\|_{W_{\infty}^{t}(\Omega)} .
$$

The inequality (4.5) now follows by summing (4.11) over $\left|\alpha^{\prime}\right|=r$ and substituting the resulting weighted semi-norm into (4.8).

We now state the corresponding result for derivatives. The proof, which follows that of Theorem 4.1, will be left to the reader.

Theorem 4.2. Suppose that the conditions of Theorem 3.1 are satisfied and in addition A.5 holds. Let $u \in W_{\infty}^{t+1}(\Omega)$ where $t$ is an integer $r \leq t \leq 2 r-2$. Let $\widehat{C}>0$ be a fixed but arbitrary constant. For any $x \in \bar{\Omega}$ let $\widehat{x} \in \bar{\Omega}$ be an arbitrary point such that $|x-\widehat{x}| \leq \widehat{C} h$; then

$$
\begin{aligned}
\left\|u-u_{h}\right\|_{W_{\infty}^{1}\left(B_{h}(x)\right)} \leq C & \left(\ln \frac{1}{h}\right)^{\overline{\bar{\ell}}}\left(h^{r-1} \sum_{|\alpha|=r}\left|D^{\alpha} u(\widehat{x})\right|+\cdots\right. \\
& \left.+h^{t-1} \sum_{|\alpha|=t-1}\left|D^{\alpha} u(\widehat{x})\right|+h^{t}\|u\|_{W_{\infty}^{t}(\Omega)}\right) .
\end{aligned}
$$

Here $\overline{\bar{\ell}}=0$ if $r-1 \leq t<2 r-2$ and $\overline{\bar{\ell}}=1$ if $t=2 r-2$.

Remarks similar to Remarks 4.1, 4.2 and 4.3 hold for the inequality (4.12). The analogue of Corollary 4.1 is the following, whose proof will be left to the reader. 
Corollary 4.2. Suppose that the conditions of Theorem 4.2 are satisfied and in addition for some point $\widehat{x}$ with $|x-\widehat{x}| \leq \widehat{C} h, D^{\alpha} u(\widehat{x})=0$ for all $r \leq|\alpha| \leq t-1$, then

$$
\left\|u-u_{h}\right\|_{W_{\infty}^{1}\left(B_{h}(x)\right)} \leq C h^{t-1}\|u\|_{W_{\infty}^{t}(\Omega)} .
$$

It is important to notice here that since $t \leq 2 r-1$, the maximal rate of convergence that can be obtained from (4.13) is $h^{2 r}-2$ which is roughly comparable to (4.7) when $t=2 r-2$.

\section{REFERENCES}

[1] H. Blum, Q. Lin and R. Rannacher, Asymptotic error expansions and Richardson extrapolation for linear finite elements, Numer. Math. 49 (1986), 11-37. MR 87m:65172

[2] Ju. P. Krasovskii, Properties of Green's function and generalized solutions of elliptic boundary value problems, Soviet Mathematics (translation of Doklady Academy of Sciences of the USSR) $10^{2}$ (1969), 54-120. MR 38:6233

[3] F. Natterer, Uber die punktweise konvergenz finiter elemente, Numer. Math. 25 (1975), 6777. MR 57:14514

[4] J. A. Nitsche, $L_{\infty}$ convergence of finite element approximations, Proceedings Second Conference on Finite Elements, Rennes, France (1975). MR 81e:65058

[5] J. A. Nitsche, $L_{\infty}$ convergence of finite element approximations, Mathematical Aspects of Finite Element Methods, Lecture Notes in Math., vol. 606, Springer-Verlag, 1977, pp. 261274. MR 58:8351

[6] J. A. Nitsche and A. H. Schatz, Interior estimates for Ritz-Galerkin methods, Math. Comp. 28 (1974), 937-958. MR 51:9525

[7] R. Rannacher, Zur $L_{\infty}$ konvergenz linearer finiter elemente, Math. Z. 149, 69-77. MR 58:8361

[8] R. Rannacher and R. Scott, Some optimal error estimates for piecewise linear finite element approximations, Math. Comp. 38 (1982), 437-445. MR 83e:65180

[9] A. H. Schatz and L. B. Wahlbin, Interior maximum norm estimates for finite element methods, Math. Comp. 31 (1977), 414-442. MR 55:4748

[10] A. H. Schatz and L. B. Wahlbin, Interior maximum norm estimates for finite element methods Part II, Math. Comp. 64 (1995), 907-928. MR 95j:65143

[11] A. H. Schatz and L. B. Wahlbin, On the quasi-optimality in $L_{\infty}$ of the $H_{0}^{1}$ projection into finite element spaces, Math. Comp. 38 (1982), 1-21. MR 82m:65106

[12] A. H. Schatz, I. Sloan and L. B. Wahlbin, Superconvergence in the finite element method and meshes which are locally symmetric with respect to a point, SIAM J. Numer. Anal. 33 (1996), 505-521. CMP 96:12

[13] R. Scott, Optimal $L_{\infty}$ estimates for the finite element method on irregular grids, Math. Comp. 30 (1976), 681-697. MR 55:9560

[14] W. Hoffman, A. H. Schatz, L. B. Wahlbin, and G. Wittum, The analysis of some local pointwise a posteriore error estimators for elliptic problems, in preparation.

[15] D. Gilbarg and N. S. Trudinger, Elliptic partial differential equations of second order, Grundlehren Math. Wiss., 2nd ed., vol. 224, Springer, 1983. MR 86c:35035

Department of Mathematics, White Hall, Cornell University, Ithaca, New York 14853

E-mail address: schatz@math.cornell.edu 